\title{
Simulation-Based Design and Rapid Prototyping of a Parallax-Free, Orthoscopic Video See-Through Head-Mounted Display
}

\author{
Andrei State, Kurtis P. Keller and Henry Fuchs \\ University of North Carolina at Chapel Hill and InnerOptic Technology Inc. \\ \{andrei|keller|fuchs\} @cs.unc.edu
}

\begin{abstract}
We built a video see-through head-mounted display with zero eye offset from commercial components and a mount fabricated via rapid prototyping. The orthoscopic $H M D$ 's layout was created and optimized with a software simulator. We describe simulator and HMD design, we show the HMD in use and demonstrate zero parallax.

\section{Introduction}

Augmented Reality (AR) applications use optical seethrough (OST) or video see-through (VST) devices. OST units are widely available as commercial off-the-shelf (OTS) products, while commercial VST-HMDs are rare.

Despite the emergence of OST-HMDs with per-pixel occlusion (e.g. [4]), VST-HMDs offer unique advantages [5]. Our group has been developing guidance systems with VST-HMDs for minimally invasive surgery. We developed a software simulator to define and optimize design parameters from which a functioning VST-HMD conforming to specifications can be built (Figs. 1-3).
\end{abstract}

\section{Previous Work}

The first stereoscopic VST-HMD we know of [6] used video cameras mounted atop an opaque HMD and had significant eye-camera parallax [5]. A newer design

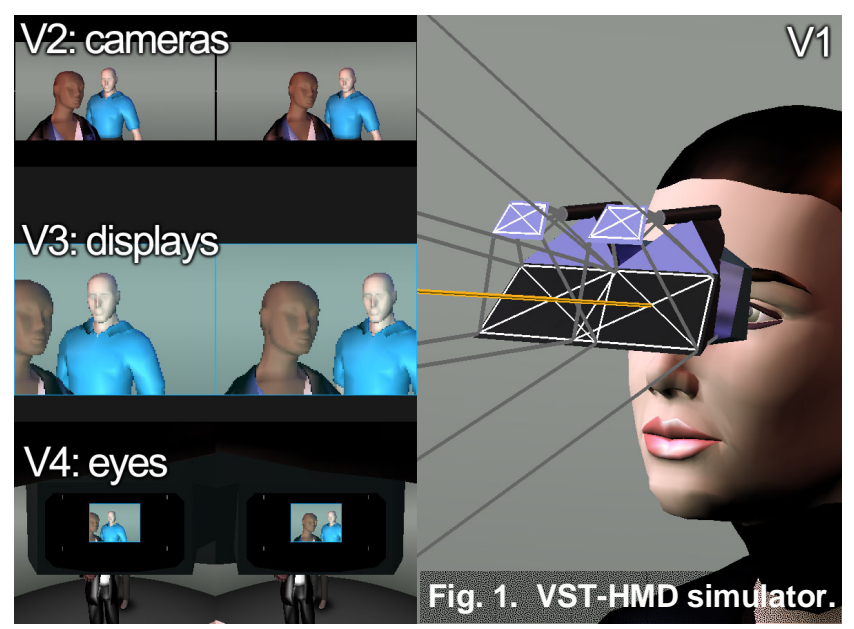

employed separate eye "pods" [2], each containing a monochrome display and a miniature camera. The camera optical path was folded with mirrors, making the device parallax-free. Since the fields of view (FOVs) of cameras and displays were matched, the device was orthoscopic [1] when properly adjusted. Another apparently orthoscopic device was used in a perceptual study [8] which led to the development of the Canon-MR Systems Lab COASTAR HMD; its optical prism lines up video cameras and display, achieving zero parallax horizontally and vertically, but not in depth-which is only essential for close-up work (e.g., in medical applications).

\section{Design Goals}

The following were our requirements for a new stereoscopic VST-HMD for medical AR work:

G1) Cameras aligned to user's eyes (parallax-free design).

G2) Orthoscopic design: peripheral imagery registered to the imagery inside the display.

G3) Camera FOV should exceed display FOV, for virtual convergence (VC) [7] and dynamic lag compensation [3]. (VC and orthoscopy can not be achieved simultaneously, of course.)

G4) "Open design" similar to earlier device [2]. The space below the display should be free of cameras or mirrors, for unrestricted downward peripheral vision.

G5) HMD should make use of OTS displays and cameras.

G6) Integrated infrared (IR) LEDs for tracking.
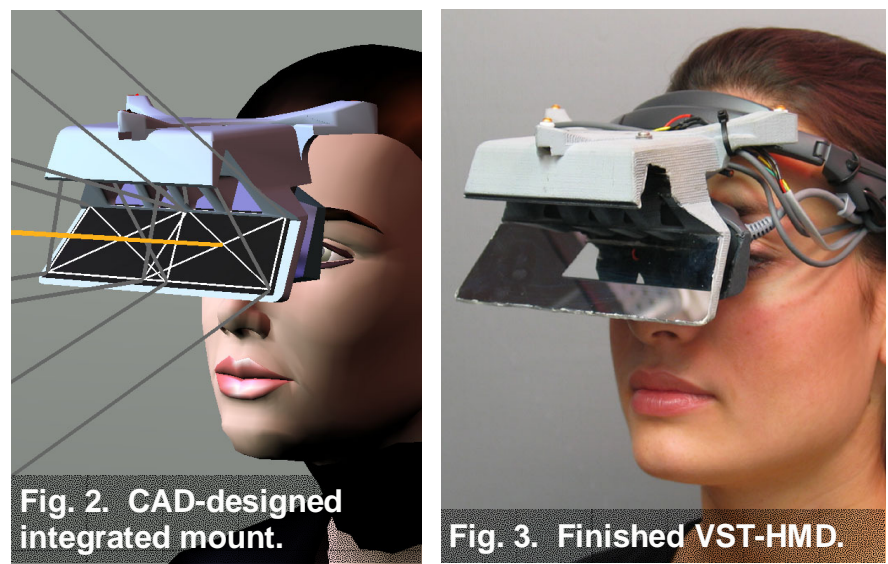


\section{The VST-HMD Simulator}

The VST-HMD simulator can interactively explore the geometric layout of cameras, mirrors and displays, as well as the kinematics of adjusting these elements to the wearer's head. We built it as an OpenGL-based program with a graphical user interface and a joystick controller. It shows a human avatar wearing a VST-HMD and uses simple pinhole optics for all calculations. The simulator presents several views to the user (Fig. 1):

V1) Entire scene: avatar, VST-HMD, and environment.

V2) Stereo imagery acquired by the simulated cameras, computed as pinpoint perspective (no lens distortion).

V3) Stereo imagery shown inside the simulated displays, derived from V2 via re-projection [7].

V4) Stereo imagery seen by avatar, with peripheral FOV.

The simulator's human avatar A (Fig. 4) has 4 degrees of freedom (DOFs):

AG) Gaze direction, implemented by rotating both eyes in their sockets (left-right, up-down, see Fig.4, top).

AC) Convergence, implemented by rotating the eyes towards or away from each other (Fig. 4, bottom).

AI) Inter-pupillary distance (IPD), realized by scaling the avatar head horizontally (Fig. 4, top vs. bottom).

Each of the simulated eye pods contains a display, a camera and either one (Fig. 5) or two (Fig. 6) mirrors that fold the camera's optical path as in the Colucci-Keller HMD [2]. The eye pod hulls morph to accommodate variations in mirror and camera pose. The pod DOFs are:
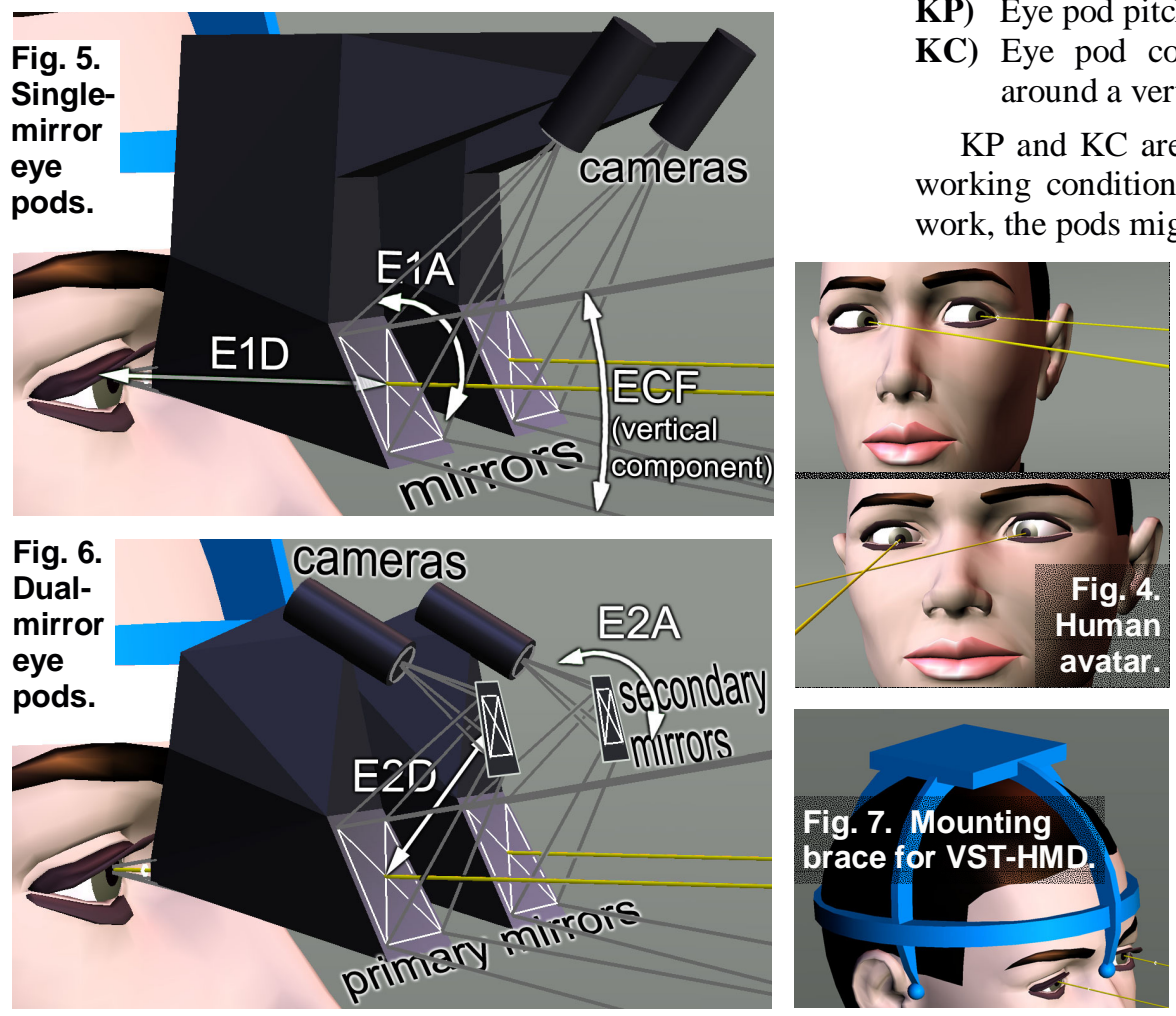

ECF) Camera FOV (vertical ECF shown in Fig. 5).

EDF) Display FOV (not shown in Figs. 5 and 6).

E1A) Primary mirror orientation.

E1D) Primary mirror's distance from the eye (to make room for the display element within the pod).

E2A) Secondary mirror orientation (if present)

E2D) Distance between primary and secondary mirrors

We simulated how an ideal VST-HMD might be donned in a repeatable way. We assumed a user-specific mounting brace $\mathrm{M}$ (Fig. 7) acting as an interface between the VST-HMD and the head. The relationship between M and the eye sockets would be the same every time a user dons his/her personal $\mathrm{M}$, leading to user-specific but constant settings for accurate alignment of the HMD with respect to $M$. In reality, a head-conforming jet pilot helmet might replace the symbolic design in Fig. 7, which uses brackets pressing against skull landmarks.

The HMD rig (Fig. 8) mates with M. It is adjusted such that the displays inside the two eye pods lie exactly in front of A's eyes and the "centers of projection" of the "mirrored" cameras lie in the eyes' nodal points, about 6 $\mathrm{mm}$ forward of the eyeball centers. Four translational DOFs serve to perform this adjustment:

KF) Forward-back motion of HMD with respect to M.

KL) Lateral motion of HMD with respect to M.

KU) Up-down motion of HMD with respect to M.

KI) Inter-display (or inter-pod) distance.

The VST-HMD rig also contains two DOFs to rotate the eye pods around the eyeball-centered axes (Fig. 8):

KP) Eye pod pitch (up-down rotation).

KC) Eye pod convergence (rotation of the eye pods around a vertical axis).

$\mathrm{KP}$ and $\mathrm{KC}$ are used to adjust for application-specific working conditions. For example, for close-up tabletop work, the pods might be pitched down and verged inward.

The following constraints were implemented to keep the simulated HMD parallax-free and orthoscopic:

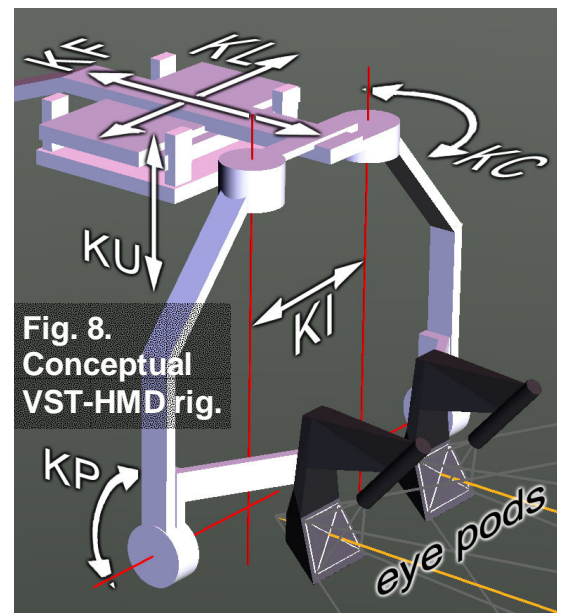


C1) If ECF is changed, the mirrors are resized to match.

C2) If E1A is changed, E2A and the cameras are rotated in order to keep the "mirrored" cameras' centers of projection in the same location.

C3) If E2A is changed, the cameras are rotated, with the same goal as in $\mathrm{C} 2$ above.

C4) If E1D is changed, the secondary mirrors and the cameras are moved, with the same goal as in $\mathrm{C} 2$.

C5) If E2D is changed, the cameras are moved, again with the same goal as in $\mathrm{C} 2$.

C6) If EDF is changed, the fragment of the camera FOV to be projected into the display [7] is resized.

Virtual convergence is controlled by a single DOF, the $\mathrm{VC}$ angle. When $\mathrm{VC}$ is active, the VST-HMD is no longer orthoscopic since the goal of $\mathrm{VC}$ is to enable stereoscopic fusion of close targets with parallel eyes.

\section{Designing a Real VST-HMD}

In view of constructing a functional VST-HMD, we prepared OTS displays and cameras. The display device available to us was a Sony Glasstron LDI-D100B HMD with stereo SVGA LCD displays (Fig. 9). Including headband, it weighs $145 \mathrm{~g}$. We measured its FOV at $26^{\circ}$ horizontal and $19.6^{\circ}$ vertical. The inter-display distance is fixed at $62 \mathrm{~mm}$, but the optics are non-pupil-forming and thus the Glasstron is suitable for users with much larger or smaller IPDs, since accurate positioning of the eye pods with respect to the eyes is not critical. We therefore decided not to build $\mathrm{M}$ nor a kinematic rig for $\mathrm{KL}, \mathrm{KF}$, $\mathrm{KU}$ and $\mathrm{KI}$ as in Fig. 8. The Glasstron head band has a double-jointed attachment which implements KP (Fig. 7). The Glasstron is built with $\mathrm{KC}=0$ (parallel displays), which is adequate for $\mathrm{VC}$ implementation.

X-ray imaging of the Glasstron (Fig. 10) revealed the option of cutting off the protruding corners, increasing downward peripheral vision. We created a 3D model of the modified Glasstron housing for use in the simulator.

We selected Toshiba IK-M43S lipstick cameras (47mm long, $7 \mathrm{~mm}$ diameter) with $4 \mathrm{~mm}$ lenses; their $50^{\circ}$ horizontal FOV is almost twice the displays' FOV. The simulator does not handle this imager's barrel distortion and will therefore yield "over-dimensioned" mirrors.

To keep the resulting VST-HMD lightweight, we enhanced the simulator with a "mirror optimization" switch that can be set to vertical-only or verticalhorizontal (V-H) cropping. The former crops the vertical extent of the primary (and secondary if present) mirrors to the vertical FOV of the displays (smaller than cameras' vertical FOV), sacrificing lag compensation for head pitch. The V-H mode (which we did not use) additionally crops the horizontal extent of the mirrors to the displays horizontal FOV, but only outwards, towards the ears, not the nose. Nasal side imagery for VC [7] is still acquired, but lag compensation for both pitch and yaw is precluded.

The following DOFs were also added to the simulator:

GP) up-down display rotation (pitch, matches KP)

GF) forward-back motion of the display (away from or towards face), adds the new constraint $\mathrm{C} 7$ below:

C7) If GF is changed, the primary mirror is moved since it cannot lie inside the Glasstron housing. Per C4, this also moves secondary mirrors and cameras.

To compensate for fabrication tolerances, measurement errors and the simple optics, we added "safety margin" DOFs that enlarge the mirrors by a few $\mathrm{mm}$ all around.

Using the VST-HMD simulator, we created and optimized a geometric layout that includes the exact positions of all components. The optimization process took several days of multi-hour interactive sessions with the simulator and group discussions. The final layout (Fig. 1) uses vertical-only mirror optimization and dualmirror pods for compactness-the cameras can be mounted right atop the Glasstron body. Since the cameras are parallel, the primary (and secondary) mirrors are coplanar and were "unified" as in [8]. This also helps ensure rigidity and symmetry.

\section{CAD, Manufacturing and Assembly}

To transfer the simulated geometry to a CAD system, we output the distances between certain selected pairs of vertices of the simulator's 3D models (19 distances in all), as well as the angles between certain vectors and planes (4 angles). These 23 numbers fully describe the VST-HMD layout. Together with measurements of the Glasstron housing, they were used to design (with a commercial CAD package) an integrated, monolithic mount holding mirrors, cameras, and IR LEDs in their simulation-derived locations (Fig. 2). To save weight while maintaining rigidity, we included numerous openings and stiffening ribs; we also added "tunnels" for the passage of wires.

The mount (Fig. 11, cf. Fig. 2) was manufactured via Selective Laser Sintering, an additive, layered rapid prototyping method. We drilled and tapped holes for the LEDs and machined away material that was obstructing the area between the camera lenses and the secondary
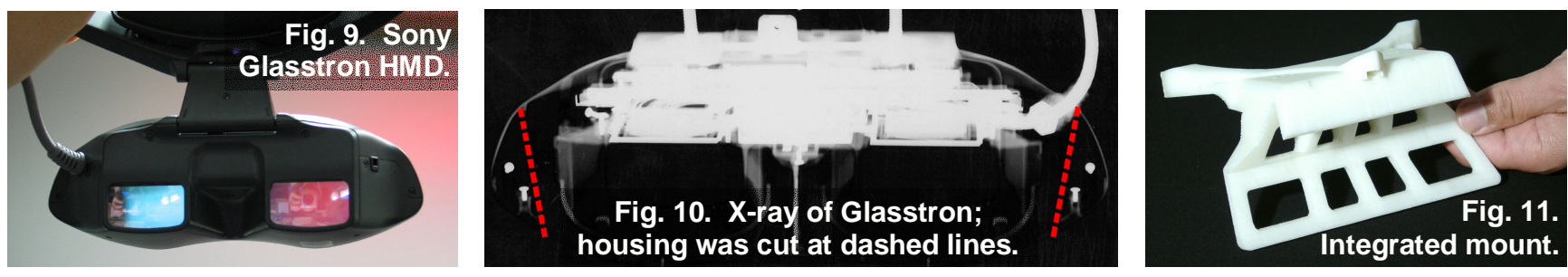
mirrors, an oversight in the CAD design. We attached mirrors, cameras and LEDs to the mount and affixed it to the Glasstron housing. The finished device (Fig. 3) weighs $330 \mathrm{~g}$ and worked on first try. We added a contoured nose pad to let it rest on the wearer's nose (the unmodified Glasstron is supported only by its head band).

\section{Using the New VST-HMD}

Integrating the new VST-HMD into our AR system (a PC port of the previously described system [6,7]) was simply a matter of calibrating the cameras as for a mirrorless HMD - the software does not to "know" about mirrors. Fig. 12 demonstrates orthoscopy and zero parallax. The HMD is only parallax-free if the wearer's IPD matches the inter-camera distance in the integrated mount, and if the wearer adjusts the HMD such that the "reflected" camera lenses are aligned with the user's eyes (that is why a head-conforming helmet would be useful).

Members of our team have used this VST-HMD for extended periods of time (often over an hour without interruption) while performing real and simulated minimally invasive procedures (cyst aspirations, biopsies, tumor ablations) on humans and phantoms (Figs. 13, 14).

\section{Conclusions and Future Work}

Using simulation and rapid prototyping, one can quickly design and build a parallax-free, orthoscopic VST-HMD suitable for demanding medical AR work. This device is likely the most sophisticated VST-HMD ever constructed and used by our team; however, we cannot yet quantify the contribution of the simulation technique, since until now this is the only device we designed using this method. A video accompanying this paper is available at http://www.cs.unc.edu/ us/VST/.

Possible improvements to the VST-HMD simulator include: simulation of camera lens distortion and design of planar mirrors with curved edges (saves weight!); more versatile eye pod design, including support for sidemounted cameras, for example; better optical models, capable of handling prisms, lenses and pupils in addition to mirrors. These will enlarge the design envelope and likely lead to improved VST-HMDs. We hope to incorporate such improvements in a future system.

\section{Acknowledgments}

We thank Jeremy Ackerman, MD (perception issues), Samuel Drake (mount fabrication), Matthew A. Mauro, MD (interventional radiologist), and Priscilla Miranda (model). Funding: National Institutes of Health (CA 47982-10).

\section{References}

[1] David Drascic and Paul Milgram. "Perceptual Issues in Augmented Reality." SPIE Volume 2653, Stereoscopic Displays and Virtual Reality Systems III (1996), 123-124.

[2] Henry Fuchs, Mark A. Livingston, Ramesh Raskar, D'nardo Colucci, Kurtis Keller, Andrei State, Jessica R. Crawford, Paul Rademacher, Samuel H. Drake, Anthony A. Meyer (MD). "Augmented Reality Visualization for Laparoscopic Surgery." Proc. Medical Image Computing \& Computer-Assisted Intervention (MICCAI) 1998, 934-943.

[3] Hiroyuki Kano, Kazuyoshi Kitabayash, Ryugo Kijima. "Reflex Head Mounted Display: HMD for Virtual Reality with Time Lag Compensation." Proc. 10th International Conference on VSMM 2004, 119-127.

[4] Kiyoshi Kiyokawa, Mark Billinghurst, Bruce Campbell, Eric Woods. "An Occlusion-Capable Optical See-through Head Mount Display for Supporting Co-located Collaboration." Proc. 2nd IEEE and ACM Intern. Symp. on Mixed and Augmented Reality (ISMAR) 2003, 133-141.

[5] Jannick Rolland, Rich Holloway and Henry Fuchs. "A Comparison of Optical and Video See-Through HeadMounted Displays." SPIE Proc. Vol. 2351: Telemanipulator \& Telepresence Technologies (1994), 293-307.

[6] Andrei State, Mark A. Livingston, Gentaro Hirota, William F. Garrett, Mary C. Whitton, Henry Fuchs, and Etta D. Pisano (MD). "Technologies for Augmented-Reality Systems: Realizing Ultrasound-Guided Needle Biopsies." Proc. SIGGRAPH '96, ACM SIGGRAPH, 439-446.

[7] Andrei State, Jeremy Ackerman, Gentaro Hirota, Joohi Lee, Henry Fuchs. "Dynamic Virtual Convergence for Video See-through Head-mounted Displays: Maintaining Maximum Stereo Overlap throughout a Close-range Work Space." Proc. Intern. Symp. on AR (ISAR) 2001, 137-146.

[8] A. Takagi, S. Yamazaki, Y. Saito, N. Taniguchi. "Development of a stereo video see-through HMD for AR systems." Proc. Intern. Symp. on AR (ISAR) 2000, 68-77.
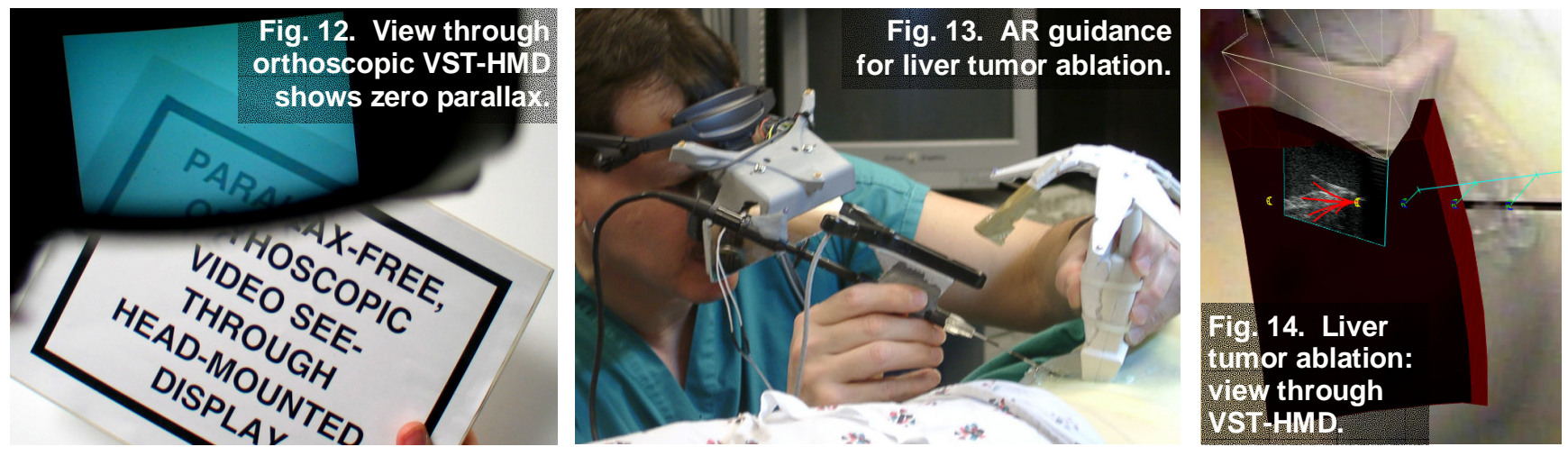